\title{
Gestión del turismo comunitario en el sector indígena de la provincia de Chimborazo caso: La Moya
}

\author{
Management of community tourism in the indigenous sector of the \\ province of Chimborazo. Case: la Moya
}

Elsa Flor Ordóñez Bravo. ${ }^{1}$, Lineth del Rocío Fernández Sánchez. ${ }^{2}$, Luis Armijo Auquilla Belema. ${ }^{3}$ \& Xavier Alejandro Silva Viteri. ${ }^{4}$

\begin{abstract}
Community tourism in Ecuador has not had the expected success due to various cultural and conjunctural factors, therefore an analysis of the management of La Moya community tourism in an indigenous sector of the province of Chimborazo was carried out, taken as a case study of the entrepreneurship of the La Moya community, whose objective was to analyze the current situation through a situational map, then a qualitative analysis was carried out that allowed determining the critical points in the processes, using the SWOT matrix. In the methodology, 35 men and women over 15 years of age who work in community tourism entrepreneurship were chosen, from whom information was obtained using tools such as situational analysis with the SWOT matrix. The results showed that the limitations of community tourism management in La Moya are of an operational and administrative nature, which leads to taking corrective actions establishing functions by job positions to avoid the diversion of material resources.
\end{abstract}

Keywords: Entrepreneurship, Tourism Management, Community Tourism

\footnotetext{
${ }^{1}$ Universidad Estatal Amazónica, Centro de Posgrado, Maestrante en Turismo, Mención Gestión del Turismo, PuyoEcuador, elsaflorob@hotmail.com

2 Universidad Estatal Amazónica, Facultad Ciencias de la Vida, Carrera de Turismo, Puyo-Ecuador, lineth1805@gmail.com

${ }^{3}$ Universidad Estatal Amazónica, Facultad Ciencias de la Vida, Carrera de Turismo, Puyo-Ecuador, luisauquilla10@gmail.com

${ }^{4}$ Instituto Superior Tecnológico Vicente León, Carrera de Gastronomía, Latacunga-Ecuador, xas337@gmail.com
} 


\section{Resumen}

El turismo comunitario en Ecuador no ha tenido el éxito esperado por diversos factores culturales y coyunturales, por ello se realizó un análisis de la gestión del turismo comunitario La Moya en un sector indígena de la provincia de Chimborazo, tomado como estudio de caso al emprendimiento de la comunidad La Moya, cuyo objetivo fue analizar la situación actual a través de un mapa situacional, luego se realizó un análisis cualitativo que permitió determinar los puntos críticos en los procesos, mediante la matriz DAFO. En la metodología se escogió 35 personas hombres y mujeres mayores de 15 años que trabajan en el emprendimiento de turismo comunitario, de quienes se obtuvo información utilizando herramientas como el análisis situacional con la matriz DAFO. Los resultados permitieron evidenciar que los limitantes de la gestión del turismo comunitario en la Moya son de carácter operativo y administrativo, lo que conlleva a tomar acciones correctivas estableciendo funciones por puestos de trabajo para evitar el desvió de recursos materiales.

Palabras Clave: Emprendimiento, Gestión, La Moya, Turismo Comunitario

\section{Introducción}

El turismo comunitario como estrategia de desarrollo es una alternativa interesante para contribuir con el progreso de las parcialidades o grupos indígenas, que por las características de sus entornos y sus manifestaciones culturales intrínsecas tienen un importante potencial para ser canalizadas como elementos para fomentar la actividad. Sin embargo, el turismo como factor productivo no se desenvuelve de forma espontánea, requiere, más que otras actividades lucrativas, una serie de elementos estructurales de índole técnico, administrativo y sobre todo relacional que le posibiliten avanzar en los procesos.

Por otro lado, el turismo no es una actividad que se despliega de forma inmediata, requiere de un largo proceso de experimentación, adaptación y fortalecimiento de los servicios, hasta cuando se haya alcanzado la medida exacta para una atención de excelencia, conjugando armónicamente los atractivos, los recursos, la infraestructura y los servicios hasta consolidarse como producto turístico.

En la provincia de Chimborazo, en las dos últimas décadas se han promovido importantes acciones para el fomento del turismo comunitario, a través de la gestión de iniciativas del Estado, los gobiernos autónomos descentralizados y organizaciones no gubernamentales, empero los resultados obtenidos de ninguna manera son alentadores, a pesar de las ingentes sumas de recursos económicos invertidos, en infraestructura, capacitación y promoción.

En este contexto, el análisis de la gestión del turismo comunitario en el sector indígena de la provincia de Chimborazo es una medida emergente, que permitirá conocer el trasfondo de la realidad de los emprendimientos turísticos y entender la problemática que surge entorno a los procesos de gestión, la difícil interacción con los organismos auspiciantes de los proyectos, así como, las complejas relaciones de poder al interior de las organizaciones campesinas, factores que 
hasta la actualidad son un obstáculo infranqueable para que los emprendimientos turísticos despeguen.

Como referente de este fenómeno se ha considerado al emprendimiento de turismo comunitario "La Moya" inaugurado en el año 2016, en la parroquia Calpi del cantón Riobamba en la provincia de Chimborazo, es una actividad turística auspiciada por el Municipio de Riobamba y la Embajada de Francia en Ecuador, con un monto de inversión superior a los \$70.000 dólares, tiene como propósito la revitalización de los saberes ancestrales, culturales y culinarios de la comunidad, con una infraestructura bastante adecuada para la actividad turística, con una aparente organización operativa y administrativa.

En aproximadamente tres años que está en operación, presenta inconvenientes que limita su consolidación como para alcanzar el nivel de producto turístico, a pesar contar con convenios y alianzas estratégicas. Además, la intrusión de la dirigencia comunitaria, falta de seriedad en los acuerdos y escaso conocimiento en gestión del turismo comunitario, paulatinamente dieron lugar al desánimo de los comuneros que iniciaron el trabajo, con las mejores intenciones de aportar a su crecimiento.

Para dilucidar los factores puntuales de la problemática del emprendimiento turístico "La Moya" se propone un análisis de gestión del turismo comunitario encaminado a determinar cuál es la situación actual de la empresa, que a partir de esta información realizar una evaluación técnica de la gestión y encontrar los puntos críticos que van a determinar las condiciones adversas que no permiten afianzarse como producto turístico.

\section{El turismo comunitario}

El turismo comunitario aparece como una alternativa para salvar las disparidades sociales y económicos que adolecen los espacios rurales, además de una vía que pueden utilizar las comunidades que buscan generar otros ingresos dentro de sus actividades económicas cotidianas, utilizando los recursos culturales, naturales y locales en una región en particular (ACS/AEC, 2014).

Este tipo de turismo permite a los habitantes de la región dada convertirse en guías turísticos y prestar servicios a los visitantes que reciben. Ofrece al propio tiempo a las comunidades la oportunidad de jugar un papel en la generación del movimiento de los elementos de producción e intercambio de bienes y servicios en la zona en cuestión (Del Carmen, 2015). Hoy en día, esta actividad sigue en constante evolución, sobre todo en los países emergentes, generando una participación más activa del turista dentro de los hábitos y costumbres de un grupo social o localidad en particular.

Según el Pan quinquenal de turismo comunitario elaborado por la Federación Plurinacional de Turismo Comunitario del Ecuador (FEPTCE, 2010), el concepto es la relación de la comunidad 
con los visitantes desde una perspectiva intercultural en el transcurso de los viajes organizados con la participación consensuada de sus miembros, garantizando el manejo adecuado de los recursos naturales, la valoración de sus patrimonios, los derechos culturales y territoriales de las nacionalidades y pueblos para la distribución equitativa de los beneficios generados.

Para la FEPTCE (2010) no es un fin en sí mismo, sino que forma parte de una estrategia más amplia tanto de desarrollo local como de consolidación política. Para las asociaciones y organizaciones indígenas y campesinas, también para las comunidades, el turismo en el que toma parte la comunidad se convierte en un medio de reivindicación y autogestión sobre sus territorios y recursos, que sirve para reclamar un lugar en el Estado y en los procesos de fortalecimiento en el ámbito productivo (Palacios C, 2016).

El turismo comunitario encierra un enfoque más participativo con respecto a la actividad turística tradicional y tiene la capacidad de convertirse en un producto turístico viable. Pero para que este potencial se pueda hacer realidad, debe existir una estrecha relación entre los planes del sector público y del sector privado, unido a las aspiraciones de las comunidades en materia de fortalecimiento del turismo en la región (Ramos F, 2016).

Los beneficios para las comunidades que participan en el impulso del turismo son varios, como la reducción de la pobreza y la generación de fuentes de empleo (Ramírez, y otros, 2016). A ello se suma que este enfoque descentraliza la industria del turismo mediante la transferencia de la toma de decisiones, contribuyendo así al diseño de productos turísticos regionales, además de conducir al fortalecimiento de la localidad, al tiempo que actúa como un estímulo para la producción de productos locales, como la gastronomía. En el Ecuador no son pocos los emprendimientos de este tipo gestados y gestionados por comunidades campesinas. Su rasgo distintivo es su dimensión humana y cultural orientada a fomentar encuentros interculturales de calidad con los visitantes. Se trata de una determinada forma de organización empresarial en el destino turístico, basada en pautas de autogestión de los recursos patrimoniales comunitarios, con arreglo a prácticas democráticas y solidarias en el trabajo y en la distribución de los beneficios generados para el bienestar de sus miembros (Rodas, Donoso, \& San Martín, 2015).

Es un modelo que se caracteriza porque las comunidades rurales indígenas o mestizas se encargan al menos de controlar una parte de esta actividad, recibiendo también ciertos beneficios económicos (Ruiz, Hernández, Coca, Cantero, \& Campo, 2008) y se entendería mejor desde la economía social y las economías populares que desde el sector turístico tradicional, ya que su elemento definitorio es su organización comunitaria.

\section{La gestión por procesos}

Desde la última década del siglo XX, la gestión por procesos ha despertado un interés creciente y en la actualidad es ampliamente manejada por muchas organizaciones que utilizan referenciales 
de Gestión de Calidad y/o Calidad Total (Ruiz F, Almaguer T, Torres T, \& Hernández P, 2014). El enfoque basado en procesos consiste en la identificación y gestión sistemática de los procedimientos desarrollados en la organización y en particular las interacciones entre tales procesos (Franch \& Guerra, 2016).

La Gestión por Procesos se basa en la modelización de los sistemas como un conjunto de procedimientos interrelacionados mediante vínculos causa-efecto. El propósito final es asegurar que todos los procedimientos de una organización se desarrollan de forma coordinada, mejorando la efectividad y la satisfacción de todas las partes interesadas clientes, accionistas, personal proveedores, sociedad en general (Martinez, 2014).

Los procesos, en este contexto, se pueden definir como secuencias ordenadas y lógicas de actividades de transformación, que parten de unas entradas (informaciones en un sentido amplio, pedidos datos, especificaciones, más medios materiales, máquinas, equipos, materias primas, consumibles, etcétera), para alcanzar unos resultados programados, que se entregan a quienes los han solicitado, los clientes de cada proceso (Sanchez, \& Blanco, 2014).

Estos procesos, al requerir un conjunto de entradas materiales e inmateriales y componerse de actividades realizadas que van transformando estos accesos, cruzan los límites funcionales repetidamente, por pasar los límites funcionales, forzando a la cooperación y van creando una cultura de empresa distinta, más abierta, menos jerárquica, más orientada a obtener resultados que a mantener privilegios (Martinez, 2014). A estas ventajas de preparación para el entorno actual, incierto y cambiante, se debe añadir la importante característica de que los procesos son altamente repetitivos. Su mejora exige una reflexión y planificación previas y la dedicación de unos medios, a veces considerables, pero proporciona un gran retorno sobre esas inversiones (Aguedo \& Escobar, 2007).

Adicionalmente es importante argumentar que las exigencias del mercado han ido poniendo de manifiesto que una adecuada gestión, que tome los procesos como su base organizativa y operativa, es imprescindible para diseñar políticas y estrategias, que luego se puedan desplegar con éxito. Con esta premisa aceptada, han surgido varios modelos de gestión basados en los procesos.

\section{La gestión y el producto turístico}

La gestión turística es el proceso integrado a través del que se gestiona cualquier tipo de destino. Abarca cuatro elementos clave: lo que ofrece el destino (la experiencia del visitante, la imagen del destino y el atractivo); la 'mezcla de visitantes' (estudios de mercado); las comunicaciones de marketing (conocimiento y promoción y la responsabilidad organizativa (liderazgo y asociación) (Navarro, 2015). 
La fragmentación ha sido una de las principales preocupaciones que impiden alcanzar efectividad en la industria turística. Con gran cantidad de instituciones públicas, a todas las escalas, y de proveedores del sector, que no entienden o no están interesados en esta dimensión del fenómeno turístico, la falta de cohesión y coordinación de los recursos es evidente y la pérdida de competitividad un hecho manifiesto. Pero, el progreso comienza con la organización de los destinos responsables para el aumento del turismo a nivel local (Pulido F \& Pulido F, 2014).

La gestión de los destinos es un tema de creciente importancia en la medida que los mismos compiten para obtener los niveles más elevados de calidad en la experiencia turística integral de los visitantes; y, necesitan gestionar los impactos del turismo en la población local y en su medio ambiente. El éxito de los destinos turísticos pasa obligatoriamente por la habilidad de gestionar todos los componentes del sistema turístico. Por esta razón se ha creado entes gestores, también denominados por la literatura especializada como Organizaciones de Gestión de Destinos (OGD) - encargados de proporcionar liderazgo en los procesos integrales de gestión (Pearce D, 2016). Este tipo de organizaciones o entes gestores han evolucionado desde una labor meramente promocional hacia entidades encargadas de liderar la gestión en todos los aspectos integrales.

Desafortunadamente no todos los destinos turísticos cuentan con una gestión que coordine todos los esfuerzos de mejora y que se ocupe de las acciones integrales al conjunto de este. En otras ocasiones dichas entidades existen, pero poseen una presencia cuasi testimonial ya que no tiene dotación presupuestaria ni personal adecuado para asumir las funciones que se le otorgan (Queiroz \& Rastrollo H, 2015). Sin embargo, la existencia de un organismo encargado de gestionar todos los aspectos integrales es absolutamente necesario bajo la realidad del mercado turístico en la actualidad cuya competitividad se intensifica de forma incesante.

La gestión adecuada debe incluir participación consensuada del sector privado y de la población local, la generación de normativa específica, transparencia en los procesos de toma de decisiones y ejecución de las acciones propuestas, respuesta efectiva ante los problemas que surjan en el día a día, equilibrio, eficacia y efectividad, gestión de los recursos financieros y visión estratégica. La gestión del destino, por tanto, se transforma en un elemento clave para garantizar el desarrollo sostenible del turismo y maximizar el rendimiento económico de este específico sector de la economía. Resulta evidente y fuera de toda duda la necesidad de contar con una gestión específica para poder garantizar el éxito del destino (Ejarque, 2016).

\section{Gestión del turismo comunitario}

La gestión del turismo comunitario responde a una visión holística que se va consolidando a largo plazo como alternativa socio-productiva que involucra a una o varias comunidades con objetivos económicos, sociales, ambientales, culturales y de sostenibilidad que están interrelacionados y son indisociables (Juarado, Domingo, \& Pastor, 2012). 
La gestión del turismo comunitario apunta a un manejo razonable de los recursos, con respeto al medio ambiente y con responsabilidad frente al entorno social (Rodas, Donoso, \& San Martín, 2015). Ese manejo se lo debe realizar de manera colectiva y no individual, para lograr un mayor y mejor control social sobre el uso de los distintos recursos, redundando en una gestión eficiente, de mayor eficacia en la adopción de decisiones y corresponsabilidad en la ejecución, seguimiento, monitoreo y evaluación del avance de proyectos y del proceso global de turismo comunitario (Palacios C, 2016).

De acuerdo con Ruiz y otros (2008), se trata pues de un ejercicio de la autogestión y de control local directo que debe cumplir con los siguientes principios fundamentales:

1. Ser socialmente solidario: Promover la cooperación efectiva entre los miembros de la comunidad y con otras comunidades, en condiciones de distribución equitativa de oportunidades y beneficios.

2. Ser ambientalmente responsable: Fomentar una conciencia respetuosa de la naturaleza y la biodiversidad con formas de gestión sostenible, vinculadas a la defensa de los derechos de uso y posesión del suelo y de los territorios ancestrales.

3. Ser económicamente viable: Incorporar objetivos e instrumentos de gestión eficaces, en el uso y valoración de los insumos que se utilizan, buscando beneficios que permitan remunerar adecuadamente el trabajo, recuperar las inversiones y lograr excedentes que se canalicen al desarrollo comunitario.

4. Ser culturalmente enriquecedor: Propiciar encuentros interculturales y experiencias de calidad, entre los visitantes y las comunidades anfitrionas, respetando mutuamente las expresiones de identidad cultural.

Pero además de lo expresado es vital comprender que la gestión del turismo comunitario involucra a varios actores del sector público, privado, comunitario y de la sociedad civil en general, entre los cuales lo ideal es crear un ambiente de confianza mutua para poder construir consensos, lazos de coordinación y la articulación necesaria para buscar el adelanto turístico local y del territorio (Ramírez, y otros, 2016).

Sin embargo en el Ecuador actual, aún se percibe desconfianza entre los distintos actores, lo que ha conducido a que sea el Estado el que pretenda generar espacios de coordinación y convergencia, aunque condicionados a un rol protagónico gubernamental que posibilite al establecimiento de directrices y políticas preestablecidas como la planificación territorial centralizada, la que aún se mantiene como rezago de la vieja planificación tradicional (Strude P, 2015). Este mecanismo no es el más recomendable dado que impide que el sector comunitario y la sociedad civil generen sus propias ideas e iniciativas de un desarrollo adaptado a los conceptos de economía solidaria y participativa. Sin embargo, también se observa que la contraparte civil y comunitaria tampoco ha adquirido la madurez requerida para crear y manejar dichos espacios de concertación. 


\section{Materiales y métodos}

La investigación se realizó en el emprendimiento de Turismo Comunitario en la Comunidad "La Moya" de la parroquia Calpi del cantón Riobamba, provincia de Chimborazo.

Macro y micro localización

\begin{tabular}{ll}
\hline Continente & América \\
\hline Subcontinente & América del Sur \\
País & Ecuador \\
Región & Sierra \\
Provincia & Chimborazo \\
Cantón & Riobamba \\
Parroquia & Calpi \\
\hline
\end{tabular}

Coordenadas:

- $749069.63 \mathrm{~m} \mathrm{E}$

- $\quad 9825182.78 \mathrm{~m} \mathrm{~S}$

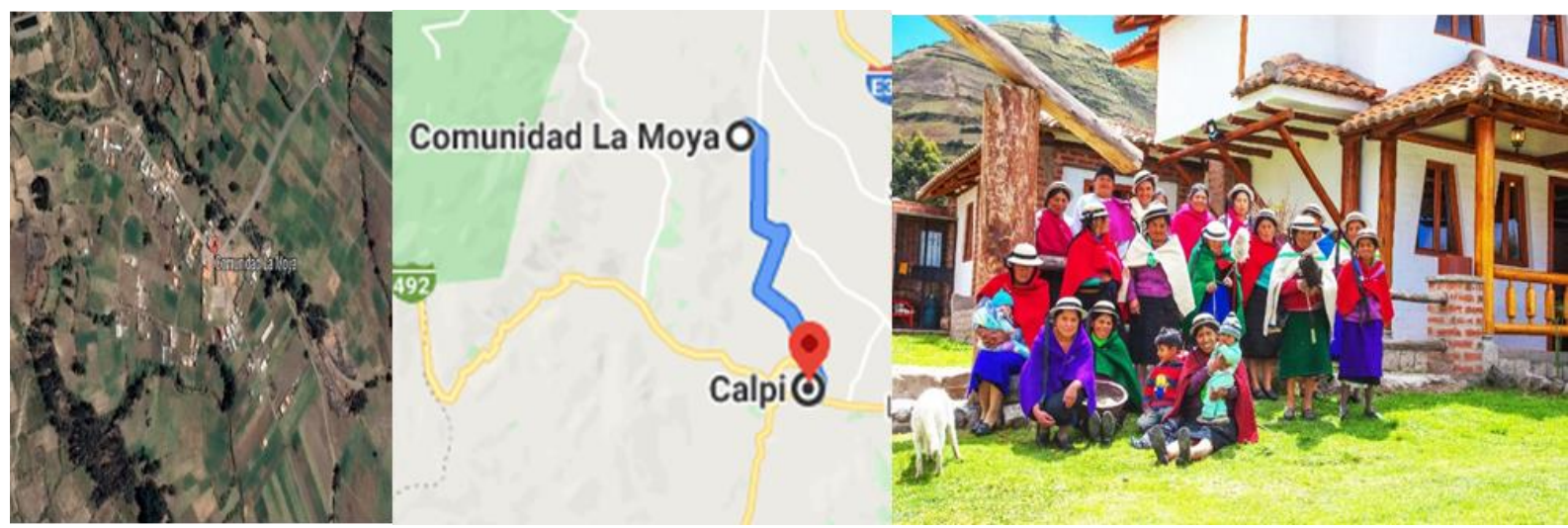

Figura 1 Ubicación de la Comunidad "la Moya" * 749069.63 m E - 9825182.78 m S

Fuente: Google Earth \& Autores

\section{Metodología}

El proceso administrativo se basa en la investigación descriptiva, que tiene por objeto establecer una descripción lo más completa posible de un fenómeno, en este caso se analizó los procesos de la gestión del turismo comunitario del emprendimiento turístico "La Moya".

Durante el proceso investigativo se utilizaron los siguientes métodos:

Método Sistémico: Es un proceso mediante el cual se relacionan hechos aparentemente aislados y se formula una teoría que unifica los diversos elementos. Consiste en la reunión racional de varios elementos dispersos en una nueva totalidad, en este sentido se hace un seguimiento a la gestión del emprendimiento de turismo comunitarios de "La Moya", se sistematizan de forma tal 
que sea visible su secuenciación y organización para luego establecer en que aspectos los conocimientos están fallando y cuáles pueden ser las posibles soluciones (Rodríguez 2017).

Histórico-Lógico: Estudia la tendencia mediante el análisis del comportamiento temporal para establecer la relación causa efecto de la gestión turística en el sector y realizar un análisis lógico de los efectos que genera su aplicación. (Rodríguez 2017).

Analítico sintético: A través del análisis de los hechos, descomponiendo en partes el fenómeno en estudio, en este caso observar las causas que no permiten realizar un eficiente modelo de gestión de turismo comunitario en "la Moya". (Rodríguez 2017)

Inductivo-deductivo: Analiza las causas particulares que no permiten una administración eficiente de los recursos en la comunidad "La Moya", establecer una ley general, a mayor despilfarro de recursos mayor problema administrativo. Parte de la Ley General llegar a conocer las causas que condujeron a establecerla.

\section{Métodos empíricos}

Bibliográfico: Donde se revisaron documentos físicos y electrónicos especializados sobre el turismo comunitario en el Ecuador, para conocer las leyes que lo rigen y su objeto de aplicación.

Observación: Parte del método científico que permitió analizar el problema en estudio para extraer su peso y establecer los objetivos estratégicos del proceso de gestión de turismo comunitario.

Se utilizó la estadística descriptiva para procesar la información recolectada y exponer los resultados en tablas y gráficos.

Cabe considerar, por otra parte, con el objeto de conocer la situación actual del emprendimiento turístico de la "La Moya" se utilizó la matriz DAFO en la que se establece por peso específico, los componentes más influyentes en el proceso para minimizar las debilidades y potencializar las fortalezas del turismo comunitario "La Moya", referente a los indicadores; administración, operatividad y promoción.

Para determinar los puntos críticos se realizó el cruce de las estrategias de la matriz DAFO, para proponer estrategias que den solución al problema.

Finalmente se aplica acciones correctivas, de acuerdo con lo identificado, para el efecto se establece funciones por puestos de trabajo estructurado de tal manera para que dé fluidez a la gestión del turismo comunitario "La Moya", considerando a los actores directos e indirectos que 
forman parte de la comunidad de acuerdo con sus fortalezas, oportunidades, debilidades y amenazas.

En el estudio se plantean las siguientes fases de la investigación mediante el análisis situacional.

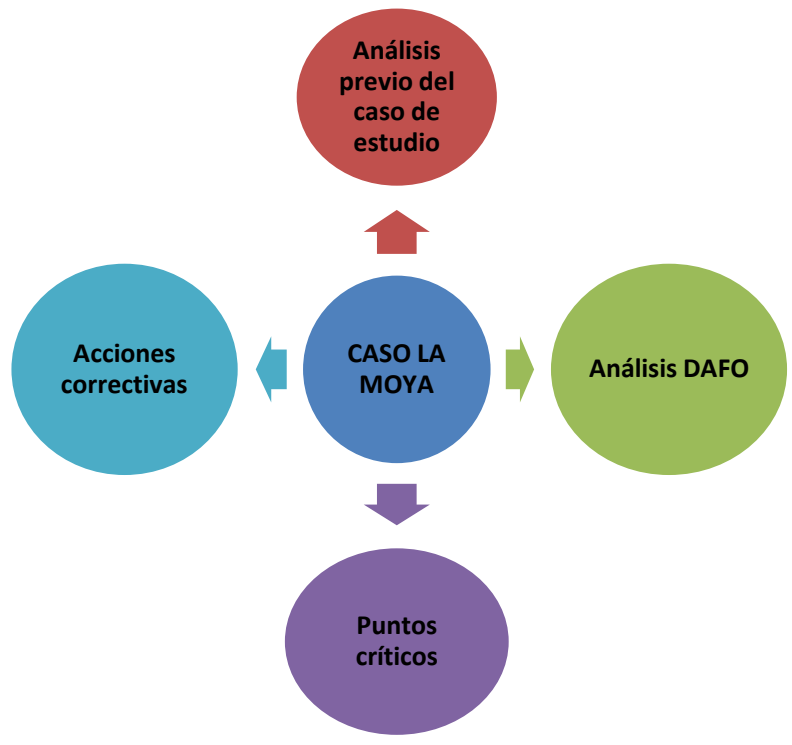

Figura 2. Fases de la investigación

Fuente: Autores

La figura 2 representa el análisis situacional del emprendimiento, diagramado en fases, que parten de investigar las circunstancias que permitieron que este caso sea considerado como un problema de investigación, seguido se representa la matriz DAFO, en la cual se establece los factores internos (debilidades y amenazas) y externos (fortalezas y oportunidades), continúa extrayendo los puntos críticos, cuyo establecimiento permite proponer las acciones correctivas que dan solución al problema de investigación.

\section{Resultados}

\section{Situación actual del emprendimiento}

Para el diagnóstico adecuado de la gestión de turismo comunitario en La Moya, se realizó un análisis situacional, en el cual se tomó especial atención en los factores internos, para establecer su peso y elaborar el nudo crítico, mediante la aplicación de la matriz DAFO.

\begin{tabular}{ll}
\hline \multicolumn{1}{c}{ FORTALEZAS } & FACTORES INTERNOS \\
\hline Casa grande & DEBILIDADES \\
Presencia del ferrocarril & Desconocimiento de administración \\
Alojamiento instalado & Exceso de personal en la cocina \\
\hline
\end{tabular}


Gran cantidad de afluentes turísticos

Amplios espacios de reunión
Inexistente organización administrativa y

financiera

Mala calidad en los servicios de alojamiento

Deficiencia en el uso del inglés

\section{FACTORES EXTERNOS}

\begin{tabular}{ll}
\hline \multicolumn{1}{c}{ OPORTUNIDADES } & \multicolumn{1}{c}{ AMENAZAS } \\
\hline Interés de las Universidades para capacitación & Desintegración de la organización \\
Interés de los turistas por conocer la zona & $\begin{array}{l}\text { Decrecimiento del afluente turístico } \\
\text { Bloqueo de carreteras por inestabilidad económica } \\
\text { Posicionamiento del turismo comunitario como una } \\
\text { estrategia de desarrollo }\end{array}$ \\
\hline
\end{tabular}

Nota: Trabajo de campo

Elaborado por: Autores

\begin{tabular}{|c|c|c|}
\hline FACTORES & FORTALEZAS & DEBILIDADES \\
\hline Externos & $\begin{array}{l}\text { 1. Casa grande } \\
\text { 2. Presencia del } \\
\text { ferrocarril } \\
\text { 3. Alojamiento instalado } \\
\text { 4. Gran cantidad de } \\
\text { afluentes turísticos } \\
\text { 5. Amplios espacios de } \\
\text { reunión }\end{array}$ & $\begin{array}{l}\text { 1. Desconocimiento de administración } \\
\text { 2. Exceso de personal en la cocina } \\
\text { 3. Desperdicio de recursos alimenticios } \\
\text { 4. No existe organización administrativa } \\
\text { 5. Inexistente área contable } \\
\text { 6. Mala calidad en los servicios de alojamiento } \\
\text { 7. Deficiencia en el uso del inglés }\end{array}$ \\
\hline \multicolumn{2}{|c|}{ OPORTUNIDADES } & AMENAZAS \\
\hline \multicolumn{2}{|c|}{$\begin{array}{l}\text { 1. Interés de las Universidades para capacitación } \\
\text { 2. Interés de los turistas por conocer la zona } \\
\text { 3. Posicionamiento del turismo comunitario como una } \\
\text { estrategia de desarrollo }\end{array}$} & $\begin{array}{l}\text { 1. Desintegración de la organización } \\
\text { 2. Decrecimiento del afluente turístico } \\
\text { 3. Bloqueo de carreteras por inestabilidad } \\
\text { económica } \\
\text { Quiebra de la organización }\end{array}$ \\
\hline \multicolumn{2}{|c|}{ ESTRATEGIAS FO } & ESTRATEGIAS DA \\
\hline \multicolumn{2}{|c|}{$\begin{array}{l}\text { F2. O2. Aprovechamiento de los recursos turísticos } \\
\text { F4. O2. Atraer turistas a la zona } \\
\text { F2. O3. Aprovechar las rutas del ferrocarril }\end{array}$} & $\begin{array}{l}\text { D1. A1. Solicitar capacitación en Administración } \\
\text { a las Universidades del sector } \\
\text { D2. A1. Organizar lugares de trabajo por áreas } \\
\text { D3. A1. Solicitar los servicios de una ecónoma } \\
\text { para evitar el desperdicio de recursos } \\
\text { alimenticios } \\
\text { D5. A2. Crear un área financiera que regule el } \\
\text { uso de los recursos }\end{array}$ \\
\hline \multicolumn{2}{|l|}{ ESTRATEGIAS DO } & ESTRATEGIAS FA \\
\hline \multicolumn{2}{|c|}{$\begin{array}{l}\text { D7. O2. Solicitar a la ESPOCH, capacitación en inglés para los } \\
\text { miembros del proyecto } \\
\text { D6. O3. Mejorar el servicio de alojamiento }\end{array}$} & $\begin{array}{l}\text { F2. A2. Implementar actividades recreativas para } \\
\text { los turistas } \\
\text { F4. A.1. Mejorar el servicio de alimentación para } \\
\text { fidelizar a los turistas }\end{array}$ \\
\hline
\end{tabular}

Nota: Trabajo de campo

Elaborado por: Autores 


\section{PUNTOS CRÍTICOS}

\begin{tabular}{|c|c|c|c|c|}
\hline \multirow[b]{2}{*}{ PUNTOS CRÍTICOS } & \multicolumn{4}{|c|}{ PRIORIZACIÓN DE LOS PUNTOS CRÍTICOS } \\
\hline & 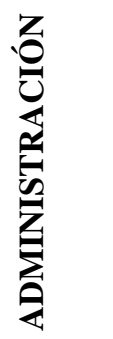 & 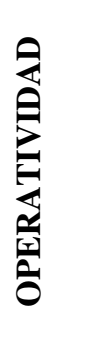 & $\begin{array}{l}z \\
0 \\
0 \\
0 \\
0 \\
0 \\
0 \\
0 \\
0\end{array}$ & 衣 \\
\hline Desconocimiento de administración & 3 & 3 & 3 & 9 \\
\hline Exceso de personal en la cocina & 2 & 3 & 3 & 8 \\
\hline Desperdicio de recursos alimenticios & 3 & 3 & 1 & 7 \\
\hline No existe organización administrativa & 3 & 2 & 3 & 8 \\
\hline Inexistente área contable & 3 & 3 & 3 & 9 \\
\hline Mala calidad en los servicios de alojamiento & 2 & 2 & 3 & 7 \\
\hline Deficiencia en el uso del inglés & 2 & 2 & 1 & 5 \\
\hline TOTAL, PONDERADO DEBILIDADES & 18 & 18 & 17 & 53 \\
\hline Desintegración de la organización & 2 & 2 & 1 & 5 \\
\hline Decrecimiento del afluente turístico & 3 & 3 & 3 & 9 \\
\hline Bloqueo de carreteras por inestabilidad económica & 1 & 2 & 1 & 4 \\
\hline Quiebra de la organización & 3 & 3 & 1 & 7 \\
\hline TOTAL, PONDERADO AMENAZAS & 9 & $\mathbf{1 0}$ & 6 & 25 \\
\hline TOTAL & 27 & 28 & 23 & 71 \\
\hline
\end{tabular}

Nota: Trabajo de campo

Elaborado por: Autores

Para priorizar los puntos críticos es necesario hacer una calificación de 1 a 3 para indicar si la variable presenta:

- Una debilidad importante (3)

- Una debilidad menor (2)

- Una amenaza menor (1-2)

- Una amenaza importante (3)

Si se toma en consideración la ponderación en forma vertical se observa que la variable operatividad tiene un peso de 28 , que es un punto crítico importante, para establecer las deficiencias, de igual forma la variable administración con un peso de 27 es de consideración frente a los 23 puntos de la variable producción.

Una vez priorizados los puntos críticos obtenidos de la matriz FODA, establecidos en las debilidades y amenazas bajo los criterios de administración, operatividad y producción se identificó los siguientes aspectos que se detallan a continuación: 
Se determino los puntos críticos a través de la sumatoria horizontal de cada uno de los indicadores, siendo los más ponderados los siguientes:

- Desconocimiento de administración puntuación 9

- Inexistente área contable puntuación 9

- Decrecimiento del afluente turístico puntuación 9

Se considero el total ponderado por indicador, el total de las debilidades es de 53 puntos, sobre el total de las amenazas 25 , lo cual significo que las debilidades se pueden fortalecer minimizando las amenazas.

\section{FACTORES CLAVE DE ÉXITO}

\begin{tabular}{|c|c|c|c|c|}
\hline \multirow[b]{2}{*}{ FACTORES CLAVE } & \multicolumn{4}{|c|}{ PRIORIZACIÓN DE LOS FACTORES CLAVE } \\
\hline & 悹 & 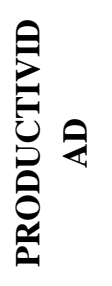 & 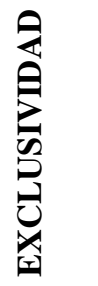 & 充 \\
\hline Casa grande & 2 & 2 & 3 & 7 \\
\hline Presencia del ferrocarril & 2 & 2 & 3 & 7 \\
\hline Alojamiento instalado & 3 & 3 & 2 & 8 \\
\hline Gran cantidad de afluentes turísticos & 3 & 3 & 3 & 9 \\
\hline TOTAL, FORTALEZAS & 10 & 10 & 11 & 31 \\
\hline Amplios espacios de reunión & 3 & 3 & 2 & 8 \\
\hline Interés de las Universidades para capacitación & 3 & 3 & 3 & 9 \\
\hline Interés de los turistas por conocer la zona & 2 & 2 & 3 & 7 \\
\hline $\begin{array}{l}\text { Posicionamiento del turismo comunitario como una } \\
\text { estrategia de desarrollo }\end{array}$ & 3 & 3 & 3 & 9 \\
\hline TOTAL, OPORTUNIDADES & 10 & 11 & 11 & 33 \\
\hline TOTAL & 20 & 21 & 21 & 64 \\
\hline
\end{tabular}

Nota: Trabajo de campo

Elaborado por: Autores

Para priorizar los factores clave de éxito es necesario hacer una calificación de 1 a 3 para indicar si la variable presenta:

- Una fortaleza importante (3)

- Una fortaleza menor (2)

- Una oportunidad menor (1-2)

- Una oportunidad importante (3) 
Para conocer los factores clave se recurre a la sumatoria horizontal de cada uno de los indicadores, siendo los más ponderados los siguientes:

- Gran cantidad de afluentes turísticos 9

- Interés de las Universidades para capacitación 9

- Posicionamiento del turismo comunitario como una estrategia de desarrollo 9

Se considera la ponderación en forma vertical se observa que la variable productividad y exclusividad tienen un peso de 21 , que es un factor de éxito importante, para establecer las oportunidades, de igual forma el peso total de las fortalezas es de 31 puntos, menor que el peso total de las oportunidades 33, lo que significa que la empresa tiene oportunidades de potencializar las fortalezas.

\section{ACCIONES CORRECTIVAS}

Una vez realizado el análisis situacional y establecido los puntos críticos, se analizó las funciones por puesto de trabajo, para el efecto se utilizó la metodología de análisis de puestos por tareas. De igual forma priorizados los factores claves del éxito determinados en las fortalezas y oportunidades bajo los criterios de calidad, productividad y exclusividad se identificó los de mayor puntuación:

- Aprovechar la gran cantidad de turistas que utilizan el servicio del ferrocarril y visitan el sector, para ofertar el servicio de alimentación.

- Posicionamiento del turismo comunitario como una estrategia de desarrollo.

Mediante el diagnóstico de la gestión del turismo comunitario "La Moya" se establece como el principal punto crítico el desconocimiento de los procesos administrativos y operativos lo que implica aprovechar sus fortalezas para potenciarlas y minimizar las amenazas que pueden hacer fracasar el emprendimiento. Del Carmen (2015), considera que el turismo comunitario permite a los habitantes del sector convertirse en prestadores de servicios y generadores del desarrollo de los elementos de producción de bienes y servicios.

Para aplicar las acciones correctivas, se estableció los objetivos estratégicos de la gestión de turismo comunitario "La Moya":

- Aprovechar el posicionamiento del turismo comunitario para impulsar el desarrollo de emprendimientos del turismo comunitario "La Moya".

- Aprovechar la gran cantidad de turistas que utilizan el servicio del ferrocarril y visitan el sector para ofertar el servicio de alimentación.

- Finalmente se aplica acciones correctivas, de acuerdo con lo identificado, para el efecto se construye un cuadro de funciones por puesto de trabajo. Para elaborar el cuadro de puestos de trabajo se utilizan varios tipos de metodologías:

\section{Análisis de puestos basados en tareas.}

En este tipo de análisis se buscó establecer las tareas que debe realizar por puestos de trabajo y sus responsabilidades (Bizneo s/f) 


\section{Análisis de puestos basados en competencias.}

En este modelo se toma en consideración las habilidades que debe tener el trabajador para desempeñar tal o cual función (Bizneo s/f)

\section{Análisis de puestos basados en fortalezas.}

Se toma en consideración el comportamiento que más les agrade realizar para lo cual es necesario estar muy motivados (Bizneo s/f)

\section{Análisis de puestos basados en tareas}

En este modelo fue necesario analizar las tareas del puesto de trabajo, para el efecto se contestan las siguientes preguntas:

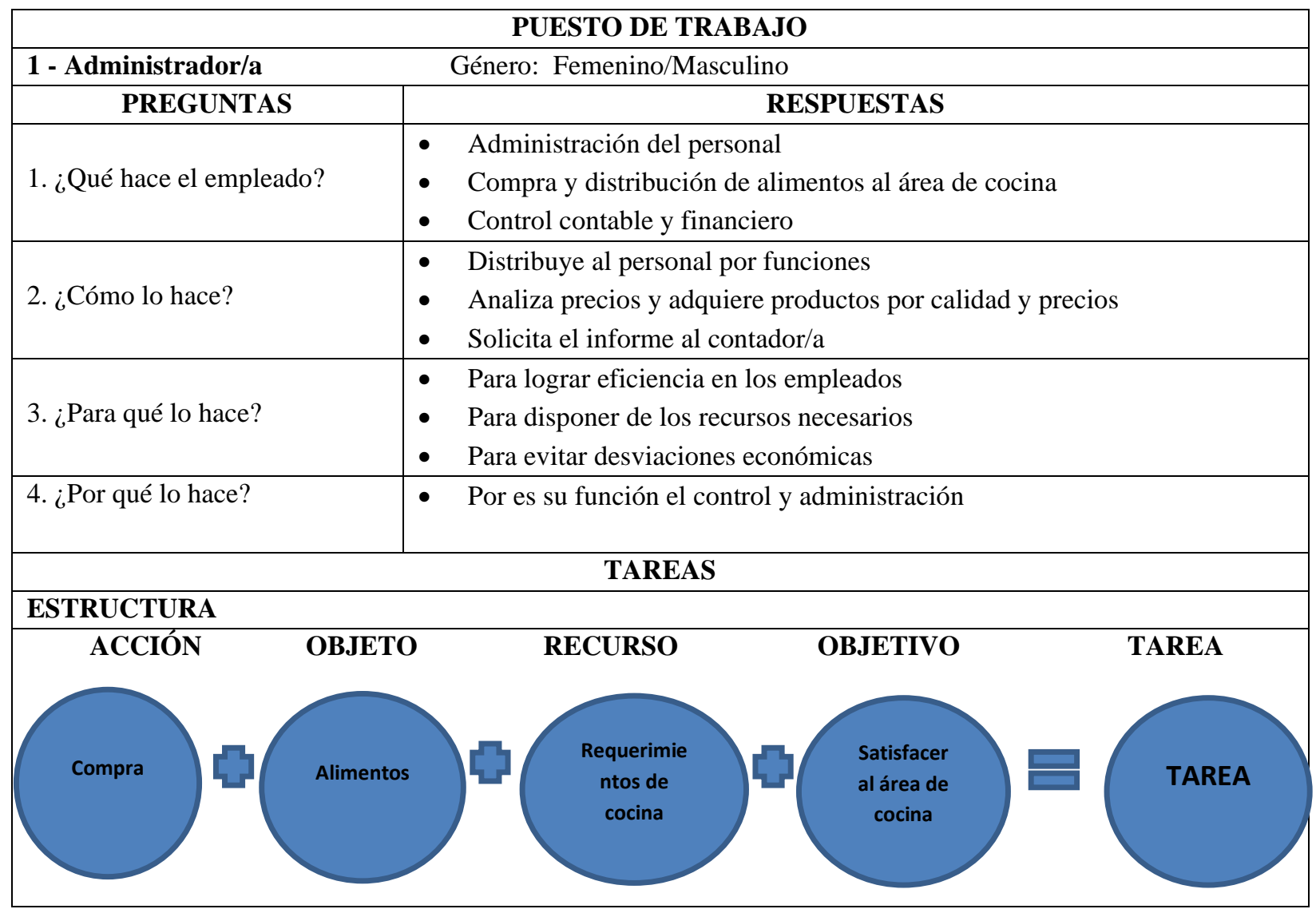

Nota: Trabajo de campo

Elaborado por: Autores

\begin{tabular}{|c|c|}
\hline \multicolumn{2}{|r|}{ PUESTO DE TRABAJO } \\
\hline 1 Contador /a & enino/masculino \\
\hline PREGUNTAS & RESPUESTAS \\
\hline 1. ¿Qué hace el empleado? & $\begin{array}{ll}\text { - } & \text { Tener listos los libros contables del emprendimiento } \\
\text { - } & \text { Establecer pérdidas y ganancias. } \\
\text { - } & \text { Llevar los libros financieros. } \\
\text { - } & \text { Presentar al administrador el estado financiero del emprendimiento }\end{array}$ \\
\hline
\end{tabular}




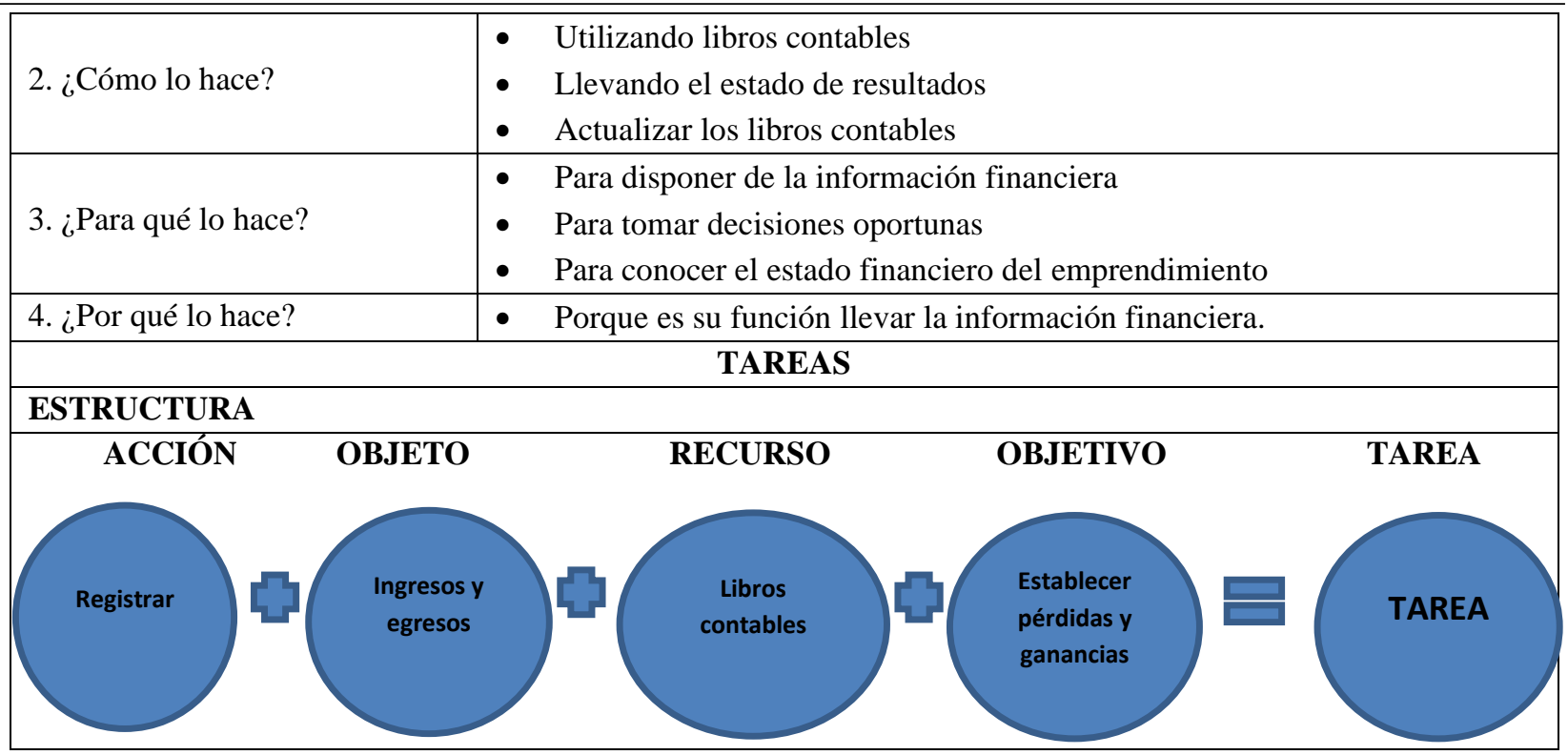

Nota: Trabajo de campo

Elaborado por: Autores

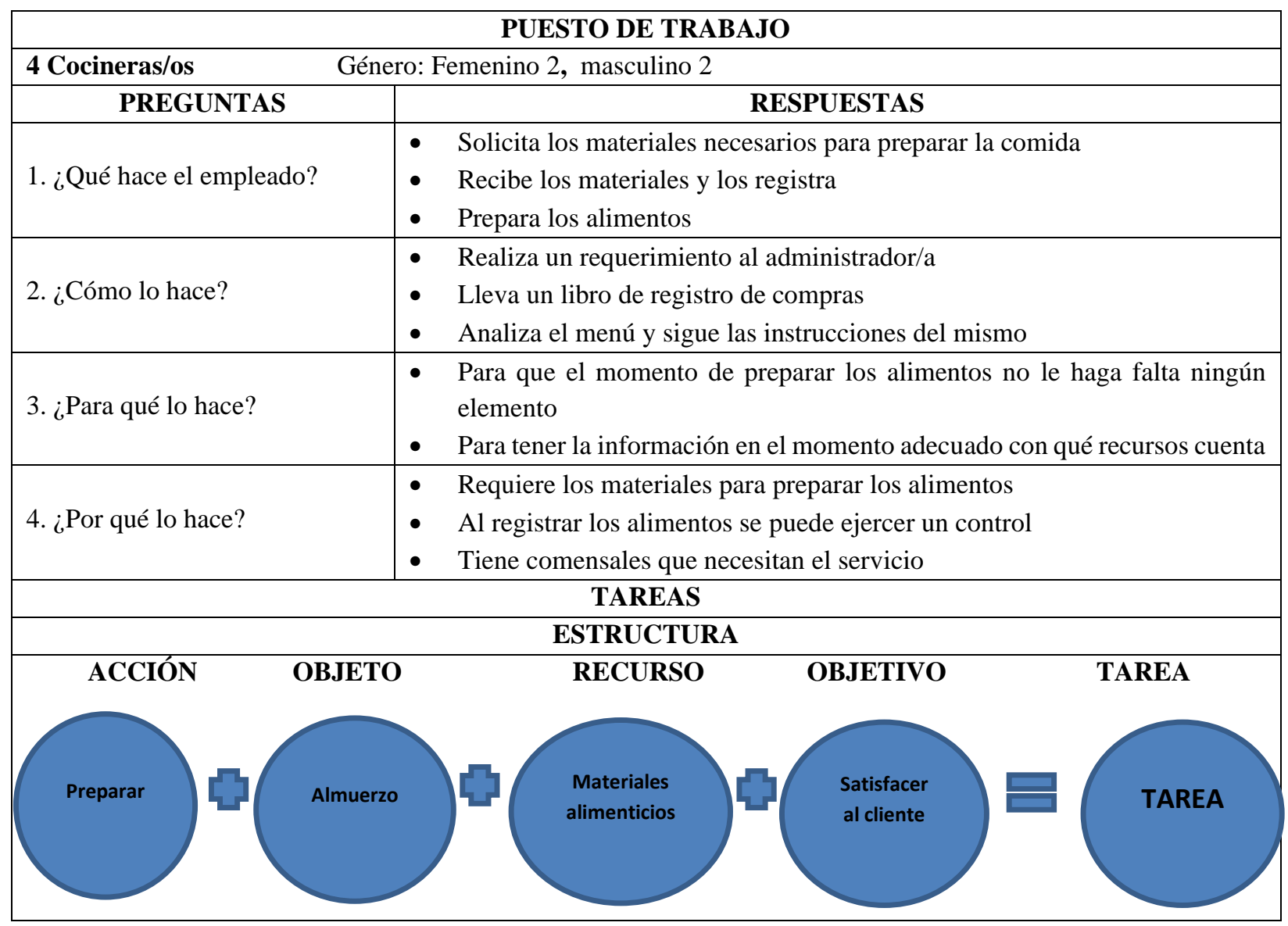

Nota: Trabajo de campo

Elaborado por: Autores 


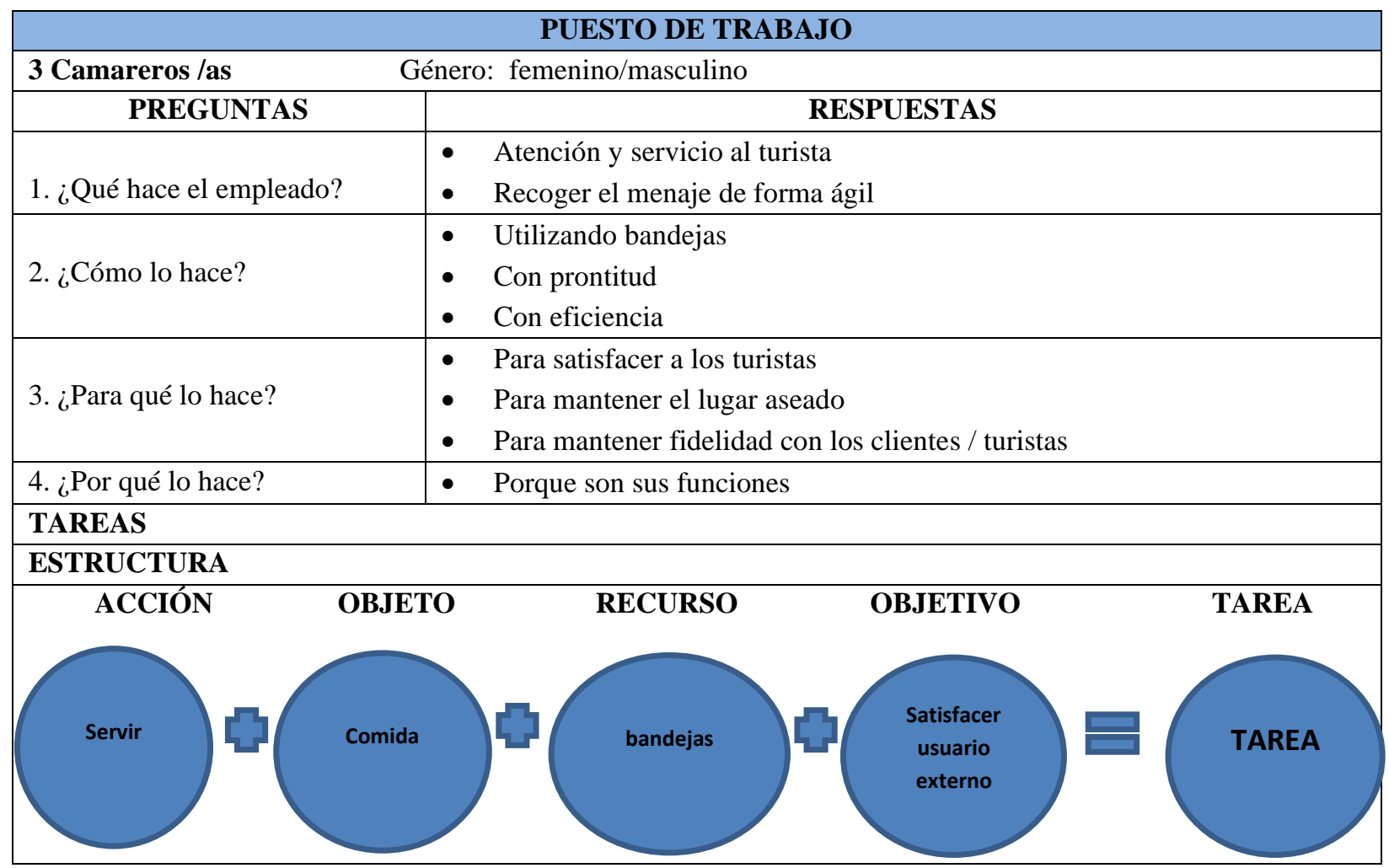

Nota: Trabajo de campo

Elaborado por: Autores

Una vez utilizado el método de tareas se tiene claro el tipo y número de personal necesario para que esta gestión de turismo comunitario "La Moya" se lleve con satisfacción para sus integrantes. Se estableció el cuadro de funciones por puesto de trabajo.

Es menester que en el emprendimiento de turismo comunitario de "La Moya" se trabaje para minimizar las debilidades como son; 1. Administración y operatividad. 2. Exceso de personal en la cocina. 3. Desperdicio de recursos alimenticio. 4. Deficiente organización administrativa. 4. Inexistente administración financiera. 5. Mala calidad en los servicios de alojamiento. 5. Deficiencia en el uso del inglés; mediante el aprovechamiento de las oportunidades como: Ventaja de las instancias públicas como Universidades y Gobierno Municipal para capacitación, Interés de los turistas por conocer la zona, posicionamiento del turismo comunitario como una estrategia de desarrollo y consolidación como producto turístico.

\section{Discusión}

La Ley de Turismo del 2002, Art. 3, literal e) establece que el "Estado reconoce la participación legítima de las comunidades indígenas, rurales, montubias, campesinas y afroecuatorianas en la prestación de servicios turísticos". En el Art. 12 se dispone que las comunidades locales organizadas y capacitadas recibirán del Ministerio de Turismo del Ecuador (MINTUR), todas las 
facilidades para el desarrollo del turismo no tendrán exclusividad de operación en el lugar que presten sus servicios. En este contexto el emprendimiento de turismo comunitario "La Moya" inaugurado en el año 2016, en la parroquia Calpi del cantón Riobamba en la provincia de Chimborazo, tiene el sustento legal necesario para realizar las actividades propuestas bajo el criterio de atención en alimentación y hospedaje con una aparente organización operativa y administrativa.

El MINTUR ha desarrollado el programa "Consolida Turismo Comunitario", que tiene por objeto mejorar el producto turístico comunitario a través del apoyo a las comunidades en asistencia técnica, capacitación, calidad y promoción; no obstante, para ser beneficiarios del programa, la comunidad debe estar; reconocida tanto por la Secretaría de Pueblos y Nacionalidades como por el MINTUR. Este apoyo del MINTUR es fundamental para la comunidad porque en el análisis situacional del turismo comunitario "La Moya" se detectaron falencias, debido a que la comunidad desconoce los procesos administrativos y operativos que permitan mejorar el producto turístico con calidad y calidez para empoderar la idea en los turistas que visitan La Moya. Se gestiona la asistencia técnica al Ministerio en el área requerida. Lo cual abaliza el análisis realizado al emprendimiento de turismo comunitario.

En el tiempo de operación del turismo comunitario "La Moya" no se ha consolidado sus servicios para que las operadoras turísticas promocionen a La Moya como un destino obligado en este sector, teniendo un decrecimiento en la cantidad de turistas que visitan la comunidad, razón por la cual se sugiere acciones de mejora para fortalecer el producto turístico, lo que significa optimizar los ingresos económicos por concepto de los servicios turísticos que ofrece la comunidad.

\section{Conclusiones}

Al culminar la investigación por parte de los autores se establece lo siguiente:

- El análisis previo del caso en estudio permitió a los autores tomar conocimiento de la situación macro del emprendimiento de turismo comunitario en la "La Moya"

- Con la identificación de los puntos críticos, se evidencio que existe desconocimiento de los procesos administrativos y operativos, inexistencia de un área contable, ausencia de registros de ingresos y egresos, para evitar el mal aprovechamiento de los recursos alimenticios y económicos.

- Al elaborar los objetivos estratégicos se determinó que es imperativo establecer las funciones por puesto de trabajo para evitar el desvío de recursos materiales como económicos. 


\section{Referencias bibliográficas}

ACS/AEC. (2014). El Turismo Comunitario . Obtenido de Asociación de estados del Caribe ACS/AEC : http://www.acs-aec.org/index.php?q=es/sustainable-tourism/el-turismocomunitario

Aguedo T, L., \& Escobar B, J. (2007). Gesti çon de procesos . Medellin: Panamericana.

Bizneo. s/f. Análisis de puestos de trabajo paso a paso. Disponible en: https://www.bizneo.com/blog/analisis-de-puestos-de-trabajo/

De la Torre P, F. (2012). Tursimo actividad Mundial. México: Trillas.

Del Carmen, R. (Dirección). (2015). Documental El Turismo y la participación de la comunidad en el siglo XXI [Película].

Ejarque, J. (2016). Marketink y gestión de destinos turísticos. s/c: Comercial Grupo ANAYA, S.A.

FEPTCE. (2010). Plan Quinquenal de turismo comunitario. Quito: Federación Plurinacional de Turismo Comunitario del Ecuador. .

Franch L, K., \& Guerra B, C. (2016). Las normas ISO 9000: una mirada desde la legislación del conocimiento, la información, innovación y el aprendizaje organizacional. Cofin HAbana, 10(2), 29-54.

Hunziker, W., \& Krapf, K. (1942). Grundriss der allgemeinen Fremdenverkehrslehre. Berlin: Polygrah. Verlag.

Juarado, A., Domingo , A., \& Pastor, V. (2012). El turismo comunitario como instrumento de erradicación de la pobreza : potencialidades para su desarrollo en Cuzco (Perú). Cuadernos de Turismo, 30, 91-108.

Martinez A, M. (2014). Gestión por procesos de negocios: Organización Horizontal. s/c: Ecobook.

Navarro, D. (2015). Recursos turísticos y attractivos turísticos: conceptualizción, clsificación y valoración. Cuadernos de Turismo, 335-357.

Palacios C, G. (2016). Turismo comunitario en Ecuador: ¿quo Vadis? Estudios y perspectivas en turismo, 25(4), 597-614.

Pearce D, G. (2016). Modelos gestión de destinos. Sintesis y evaluación. Estudios y Perspectivas en Turismo, 25(1), 1-16.

Pulido F, M., \& Pulido F, J. (2014). ¿Existe gobernanza en la actual gestión de los destinos turísticos? Estudio de casos. Pasos, Revista de Turismo y Patrimonio Cultural, 12(4), 685-705.

Queiroz, F., \& Rastrollo H, M. (2015). El estado del arte en gobernanza de destinos turísticos. Tourism \& Management Studies, 11(2), 47-55.

Ramírez, O., Paúl, H., Velíz, N., Tania, I., Roldán, A., \& Ferrales, Y. (2016). Emprendimiento como factor del desarrollo turístico Rural sostenible. Retos de la Dirección, 10(1), 71-93. 
Ramos F, M. (2016). Análisis de las políticas para el turismo en Ecuador con enfasis ene 1 turismo rural comunitario. Revista San Gregorio, 15, 122-127.

Real Academia de la Lengua Española. (2014). Disccionalrio de la real Academia de la Lengua Española (Vigesima tercera edición ed.). Madrid: Espasa.

Rodas, M., Donoso, U., \& San Martín, I. (2015). Turismo Comunitario en el Ecuador: Una revisión d Literatura. Revista de Investigación de la Ciencia Turística - RICOT, 9, 60-77.

Rodríguez, A. y Pérez, A. O. (2017). Métodos científicos de indagación y de construcción del conocimiento Revista EAN, 82, pp.179-200. https://doi.org/10.21158/01208160.n82.2017.164

Ruiz B, E., Hernández, M., Coca, A., Cantero, P., \& Campo, A. (2008). Turismo comunitario en Ecuador. Comprendiendo el community-based tourism desde la comunidad. Pasos, Revista de turismo y patrimonio cultural, 6(3), 399-418.

Ruiz F, D., Almaguer T, R., Torres T, I., \& Hernández P, A. (2014). La gestión por procesos, su surgimiento y aspectos teóricos. Ciencias Holgín, 20(1), 1-11.

Sanchez, , L., \& Blanco, B. (2014). La Gestión por procesos un campo por Explotar. Dirección y Organización, 54, 54-71.

Strude P, C. (2015). Modelo de Gestion participativa del desarrollo turistico comunitario en la parroquia de LLoa. Quito: PUCE. 


\section{PARA CITAR EL ARTÍCULO INDEXADO.}

Ordóñez Bravo, E. F., Fernández Sánchez, L. del R., Auquilla Belema, L. A., \& Silva Viteri, X. A. (2020). Gestión del turismo comunitario en el sector indígena de la provincia de Chimborazo caso: La $\quad$ Moya. $\quad$ Explorador $\quad$ Digital, https://doi.org/10.33262/exploradordigital.v4i4.1412

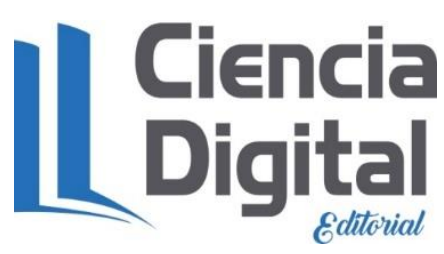

El artículo que se publica es de exclusiva responsabilidad de los autores y no necesariamente reflejan el pensamiento de la Revista Explorador Digital.

El artículo queda en propiedad de la revista y, por tanto, su publicación parcial y/o total en otro medio tiene que ser autorizado por el director de la Revista Explorador Digital.
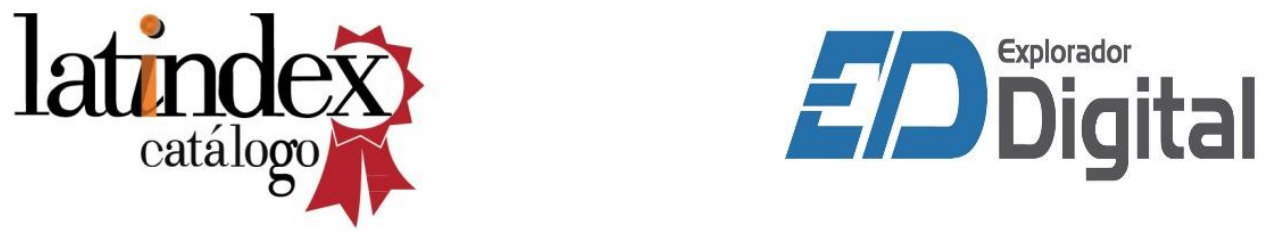